\title{
Paradigmatic shifts in occlusion and temporomandibular disorders
}

\author{
M. M. ASH University of Michigan, School of Dentistry, University of Michigan, Ann Arbor, MI, U.S.A.
}

SUMMARY There are several terms that identify proposed paradigms for the way things ought to be carried out in the health sciences: evidence-based, cause-and-effect, diagnostic gold-standard, patientcentred-outcomes, risk assessment, cost/benefit/ risk, and efficacious/effective. Collectively these paradigms exhibit varying degrees of interdependence, and have the potential for changing the way dentistry is practiced. A paradigm can be thought of as a standard by which research and health science ought to be conducted and evaluated. In this sense scientists and clinicians try to figure out how to account for various observations and phenomena dictated by paradigms or models of health care; however, it may become necessary to shift to new paradigms that are more consistent with scientific and clinically reality. Some of the potential effects of these shifting paradigms on the practice and teaching of occlusion and temporomandibular disorders are considered.

KEYWORDS: evidence-based, occlusion, paradigms, TMD

\section{Introduction}

The sine qua non of ongoing paradigmatic shifts is a movement from the 'art and science' of dentistry, which is said to be based on biological/mechanical knowledge, towards a practice hopefully based at best on scientific, quantitative data from controlled, randomized clinical trials. This information transfer is sometimes viewed as a challenge to the dental profession in that it questions what and how practitioners know what they practice is truly efficacious. The resulting controversies have been referred to as a 'clash of culture' between researchers and clinicians (Raphael \& Marbach, 1997). However, some of the controversies over occlusion seem to suggest deep-seated ideological needs to champion particular points of view that are based more on theory-laden ideas than on truly, theory-neutral observations. Presuppositions pervade every aspect of human activity, science (and scientists) being no exception (Casti, 1989), which is the nucleus of the idea underpinning Thomas Kuhn's notion of a scientific paradigm (Peat \& Briggs, 1984; Kuhn, 1996).
Irrespective of the pros and cons of the relationship of occlusion to temporomandibular disorders (TMD), some of the controversial positions taken appear to be so coloured by perceptual and ideological presuppositions that each one conditions its own observational evidence. In the absence of truly theory-neutral observations and observers, one paradigm is assumed to be as good as another. In this respect, several paradigms can be supported by the same evidence (Butts, 1995). If so, it is possible to agree with Kafka in The Castle: 'Nowhere in the castle of science is there a final exit to the absolute truth.' (Kafka, 1954; Rucker, 1982).

\section{Evidence-based health care}

Evidence-based health care can be considered to be 'conscientious, explicit, and judicious use of the current best evidence in making decisions about the care of individual patients ... including integrating individual clinical expertise with best available external clinical evidence' (Sackett et al., 1996). Thus, it is the practitioner with the clinical experience, judgement, 
and knowledge of the individual patient's needs who actuates evidence-based health care in the long term theatre of clinical reality. Without this transfer of evidence-based information from researcher to clinician, evidence-based health care is problematic. However, for a meaningful transfer of information to be successful, both research and health care must be evidencebased. In order to make science clinically relevant, the transfer of information between research and practice must be bi-directional. Only then can the gaps between what is known and what is practised be reduced in a meaningful way.

The evidence-based paradigm argues for the primacy in dental practice of probabilistic knowledge derived from clinical studies using statistical methods. Thus, researchers propose to provide a degree of certainty for what is probable into a world that may reflect varying degrees of clinical uncertainty. Perhaps, it is thought that clinicians should implement the findings from probabilistic research into clinical practice automatically; however, it is quite possible that the inferential leap needed to treat an individual patient based on aggregate findings cannot be assumed. Clinicians do not always relate to probabilistic research findings; they sometimes need to access their own store of knowledge to deal with the complexity of clinical experiences involving the immediacy and individuality of patients in clinical reality. Practitioners may not follow the rules of a paradigm per se; they intuit what is right and appropriate, including that it is sometimes right to defer to a rule (Tanenbaum, 1999).

The researcher's challenge to clinicians to provide evidence-based health care requires clinicians to challenge researchers to use evidence-based methodology and truly theory-neutral interpretation of their research data. The ultimate goal for research and for clinical practice is to be evidence-based; however, that cannot be forced by simply putting 'The Emperor's New Clothes' (Andersen, 1974, 1997) on occlusion and TMD and muscle disorders (Kirveskari \& Alanen, 1999). Obviously, the evidence-based paradigm has considerable interest for insurance and governmental cost containment strategies (Davies, 1999).

It is unlikely that any practitioner would reject in theory the concept of evidence-based dental treatment. However, the evidence against a generally accepted form of treatment must be based on high levels of evidence quality and not simply on differences be- tween statistical and biological significance (Pincus $\delta$ Stein, 1999) that may not be a reflection of clinical reality. Therefore, although experiments conducted as randomized clinical trials have been scientifically helpful, improved methodology will have to include evidence obtained in the unplanned 'experiments' of ordinary clinical practice (Feinstein, 1983a,b,c,d).

For some, evidence-based health care is conceptually not new and as now proposed, is nothing but the thinly disguised worship of statistical methods and techniques (Boa, 1998), new priority for an old paradigm (Feussner, 1996), or popular nonsense, old wine in new bottles, or current necessity (Raspe, 1996). In effect, such thinking suggests that an evidence-based paradigm represents a way to reduce the costs of health care for the benefit of patients, insurance companies, and governmental agencies. However, the foundation for the evidence-based paradigm rests on the validity of other paradigms, not just cost/ benefit/risk formulas for health care. Practitioners do not reject an evidence-based paradigm when it reflects a sense of clinical reality.

\section{Evidence-based research}

The guidelines for evidence-based recommendations should come from clinical trials and, where evidence is lacking or absent, should reflect the considered opinions in the field (Agency for Health Care Policy and Research, 1992). Longitudinal data is often collected in clinical trials to examine the effect of a treatment on a disorder over time (Bellamy, 1999). However, it cannot be assumed that the effects of treatment reflect a direct affect on the cause of the disorder unless its cause is known and additional evidence exists to demonstrate a direct cause-and-effect relationship. Therefore, most clinical trials are comparisons of the effects of an active therapy versus a placebo, assuming that a difference between the two in favour of the active therapy reflects an effect on the cause of the disorder. That conclusion may not be true, especially when the cause of the disorder has not been established.

The random allocation of treatment in clinical trials reflects an unpredictability that reduces susceptibility to bias in treatment groups assigned preferentially according to prognostic differences. Even so, prognostic analyses are needed for evaluating imbalances in randomization, for precise clinical application of the re- 
sults, and for discerning disparate therapeutic effects (Feinstein, 1983a,b,c,d).

It cannot be concluded that all evidence is based on valid methodology and interpretation. Therefore, the paradigm evidence-based should be applied to research as well as to health care. For example, it would be a misperception to assume that all current methods of statistical inference are all evidence-based. Without biological plausibility and prior evidence, statistical methods cannot provide a number that by itself reflects the probability of reaching an erroneous conclusion. Thus, it cannot be assumed that a single number, e.g. $P=0.06$, can capture both the long-run outcomes of an experiment as well as the evidential meaning of a single result (Goodman, 1999a). Questions about the appropriateness of statistical methods used in clinical trials are not new (Davidoff, 1999; Goodman, 1999b); inconsistencies are not limited to dental science (Rothman, 1986).

Some of the controversy about evidence-based treatment and procedures exists because research findings and their interpretation have not met the highest levels of evidence quality, e.g. not based on meta-analysis of randomized controlled trials (Agency for Health Care Policy and Research, 1992; Niederman, 1998; Palmer \& Sendi, 1999), or at least one randomized control trial like that of Kirveskari et al. (1998) and Burgett et al. (1992). Often the data being used to support or reject occlusal therapy for 'TMD' is based on the lowest quality level of evidence, i.e. from expert committee reports or opinions and/or clinical experience of respected authorities (National Institute of Health Technology Assessment Conference Statement, 1996). Of course the research methods underlying randomized clinical trials must first meet certain diagnostic and treatment standards. All research can be improved upon, and critiques of research findings can lead to better research methods and improve the interpretation of research findings. It is sometimes assumed that casual inference exists between two variables when all spurious associations have been ruled out. When that unlikely event occurs, the probability of a causal relationship may be there on an epidemiological basis but other evidence is required, e.g. placement of occlusal interferences that cause traumatic TMJ arthritis. In addition, the effect-effect relationships are difficult to assess without knowing all possible cofounders and testing for an association. Unfortunately, it is much easier to deny a cause-and-effect relationship based on the limitations of a study than to design and carry out studies to prove or disprove that cause-and-effect relationships exist between occlusion and 'TMD'

Research and review articles may include support for only one point of view without citing valid evidence for other positions. It might be expected that critiques of research data would undertake meta-analysis of independent studies to evaluate their results collectively (Normand, 1999), and use Bayesian persuasion probabilities that should persuade the a priori of most opinionated parties to change their views (Everitt, 1998). However, meta-analysis and the Bayesian techniques are little used in dental research (Jakobsen, 1999). Reluctance to use these methods may occur because of the limited number of controlled, randomized clinical dental trials that are available and appropriate, knowing what trials to include, to what population the results actually apply (Everitt, 1998), and because beginners may hesitate using these methods (Moore, 1997). Even so, the quality of evidence is considered to be highest when the use of meta-analysis is valid and possible. It is unlikely that researchers or clinicians would be opposed to evidence-based research methods, including appropriate statistical methods to evaluate the results of several clinical trials.

Controlled, randomized clinical trials remain the standard for evaluating one therapy versus another or placebo (Feinstein, 1983a,b,c,d). Such trials are most effective for acute diseases, but several limitations are present in trials of chronic disorders, e.g. the problems of patient selection, exclusion criteria, relevance of surrogate markers for long term outcomes, placebo effect, and the problem of applying the difference between what is statistically and biologically significant. In retrospect, many studies exhibit some of these problems, including disclaimers that another study is necessary. The requirements for randomized clinical trials are not always met (Chalmer et al., 1981), and the results of such studies may be the basis for unyielding different points of view.

\section{Cause-and-effect paradigm}

A cause is a condition that is sine qua non for the occurrence of an effect. This definition can be the basis of a cause-and-effect paradigm in which both the cause and the effect are parsed, e.g. causes may be unknown 
or multifactorial and effects may multifarious. In effect, causes may be viewed as being probabilistic and the collective effects expressed as 'TMD'. Perhaps the term 'TMD' can be viewed as a meme or memeplex, i.e. elements of culture passed on by non-genetic means (Dawkins, 1989; Blackmore, 1999). Given that the paradigm may reflect more than one concept, it has an inferential meaning that, in order for a clinical treatment or a procedure to be accepted as evidencebased', appropriate research evidence must demonstrate that it has an effect on the cause of the disorder, not simply relief of symptoms or a placebo effect. Such therapy must have a highly predictable and consistent effect on the cause of the disorder. This cause-and-effect paradigm makes specific treatment for the causes of diverse TMD problematic when the aetiology of 'TMD' is considered to be unknown (Merskey \& Bogduk, 1994) or multifactorial (DeBoever, 1973). In effect, if the cause(s) of a multifarious disorder such as 'TMD' remains undetermined, it is not possible to specifically treat an unknown aetiologic factor, or to use a 'shotgun' approach to address a subset of disorders having questionable multifactorial aetiologies. In addition, the probability of a single aetiologic factor being the cause of 'TMD' or any oro-facial disorder is generally considered to be remote (Spilker, 1991). If it is considering that the cause of each case of TMD is multifactorial, the causal factors must be considered to form a composite cause. The paradigm of composite causes is not reconcilable with parsing percentage contributions to the composite cause because they reflect a total of infinity, not an assumed $100 \%$ (Rothman $\delta$ George, 1998; Kirveskari \& Alanen, 1999).

It is difficult, if not impossible, to establish with one $100 \%$ certainty a cause-and-effect relationship between 'TMD' and bruxism (Lobbezoo \& Lavigne, 1997). The same may be said about the relationship between occlusion and 'TMD'. Therefore, until 'science' has determined the specific causes of 'TMD' in its various forms, the practitioner must utilize an occlusal therapy that is consistent with that level of evidence available to support the concept that occlusal therapy has a supporting role in the treatment of 'TMD' and occlusal dysfunction. For example, in cases such as, bruxism and clenching, trauma from occlusion, progressive periodontitis, and interferences to occlusal function.

\section{Diagnostic gold standards}

A diagnostic 'gold standard' for a disorder or a disease is the consensus definition of what constitutes a disorder or disease, e.g. criteria developed by the American Academy of Orofacial Pain (1996), and the International Headache Society (1988). The widely accepted single 'gold standard' for 'TMD' includes: pain in $\mathrm{TMJ}(\mathrm{s})$ and muscles of jaw movement, limited range of movement (ROM), and clicking and crepitus in the joints (Storey, 1994). The initial treatment of 'TMD' calls for conservative, reversible therapy, often based on the findings of the single gold standard that may point to a spectrum of disorders not necessarily having the same aetiology. Although the success rate for the conservative treatment of 'TMD' appears to be high, there are legitimate questions by critics about the possibility that the treatment is largely placebo and recovery would have occurred without treatment. Perhaps true, but how soon this might occur varies from weeks to years, which may not equate with patient-centred criteria. There are a small percentage of the 'TMD' patients, perhaps $15 \%$ that have persistent problems, especially pain. Diagnostic protocols to identify these patients with more complex 'gold standards' include the dual-axis diagnostic protocol (Dworkin \& LeResche, 1992), TMJ Scale (Lundeen et al., 1986), and the Craniomandibular Index (Fricton \& Schiffman, 1987). However, all indices have their advocates and critics, and all have their limitations. What is needed is the adoption of diagnostic criteria that allow both clinicians and investigators to identify comparable subsets of the TMD spectrum (Storey, 1994), perhaps something like the clinical diagnostic operational and optional criteria proposed by Truelove et al. (1992). It also has its critics who disagree, for example, with the idea that a symptom can be a diagnosis.

\section{Patient-centred outcomes paradigm}

An assessment of the success of dental treatment, or the usefulness of dental procedures, has been based largely on what the practitioner perceives to be true, e.g. personal satisfaction with the application of occlusal schemes said to improve function, increasing mouth opening to a standard of $40 \mathrm{~mm}$, and obtaining a balanced denture occlusion. These outcomes, and a number of other expressions of success, often do not 
reflect patients' perspectives of successful treatment. It is not implied that the practitioner's assessment is incorrect; however, it does suggest that there is a shift toward patient-centred criteria to include those areas of health care identified to be of value to the patient, i.e. toward what is considered by the patient as well as health care managers to be 'health-gain' (Davies $\delta$ Crombie, 1997). Although the practitioner may not be able to justify the biological advantage of an occlusal scheme on the basis of what is considered to be 'scientific' evidence at the time, the use of patient-centred outcome criteria may satisfy the requirements for an evidence-based paradigm, i.e. studies that show effectiveness based on patients' perspectives of the outcome of treatment (Dao \& Lavigne, 1998), and their requests for treatment (Kirveskari et al., 1998). Criteria from research on patient-centred outcomes have been used in the medical field (Gerszten, 1999; Tanenbaum, 1999), and appear to be the direction for some dental studies (Feine et al., 1997; Kirveskari et al., 1998).

Some of the research cited by critics of occlusal therapy for 'TMD' conclude that therapies used are frequently no better than a placebo. The pros and cons of that kind of critical assessment fuel the fires of controversy. However, a paradigm shift to scientifically derived patient-centred outcomes criteria may be used to redirect the apparent lack of correlation between what dental practitioners perceive to be the criteria for successful therapy, and what patients consider to be satisfactory treatment. For example, the majority of patients with dentures are satisfied irrespective of the occlusion of their denture, i.e. chewing efficiency and patients' satisfaction appear to be more important to the patient than denture occlusion (Palla, 1997). Of course, that is not much different from what Stallard (1965) said almost 40 years ago: ‘... balanced occlusion is not suitable for the natural teeth, and probably not fit even for denture teeth'. Recent research suggests that cuspid guidance in dentures is better than group function. However, it should not be concluded that denture occlusion is not important. The occlusion must satisfy to the extent possible, both mechanical principles and positioning of the dentures in the functional space, including freedom in centric, centric relation and individualized tooth arrangement (Palla, 1997). As already suggested, professional knowledge blended with an appropriate addition of patient-centred criteria may be the experiential mix needed for clinical reality and an evidence-based practice.

\section{Risk assessment paradigm}

Studies about the assessment of risk factors to determine what patients are more or less likely to prevent or control are more often seen in the areas of caries and periodontal diseases than occlusal and TMD. Risk factors are viewed as being conditions, behaviour, and patient characteristics that tend to be associated more frequently with dental disease or disorders. The concept can extend to bruxism and clenching and other parafunctional activities. Risk assessment should be one of the basics of evidence-based practice. The absence of significant concerns for risk assessment relative to occlusal and TMD is puzzling, but not unexpected. Risk assessment does not usually consider dentists but could extend to that dimension ultimately.

Studies of attrition generally relate to changes in the occlusion with age, function, restorations present, bruxism, and 'TMD' (Beyron, 1954; Molnar et al., 1983; Hugoson et al., 1985, 1988; Dahl et al., 1989; Seligman \& Pullinger, 1995). The results of these studies suggest that individuals in general may maintain a low level of wear over their lifetime while others show very high levels very early in life.

There is little information that can be used as a risk proxy for aggressive parafunction wear. Perhaps dentists should include in their examination of young adult patients some index of occlusal wear or make casts of their occlusion periodically to follow the character and progress of wear. It is unlikely that third party payers would be interested in underwriting the cost. However, where there already exists evidence of moderate to severe bruxing and clenching, the dentist needs to consider the risk of not providing preventive measures, e.g. in selective occlusal adjustment to reduce excessive forces on susceptible teeth, proper restorative design (e.g. onlays versus inlays), and use of stabilization type occlusal bite plane splints.

Many problems raised by the following questions have not been resolved by evidence-based research. What is the probability that early occlusal adjustment of the teeth, especially those with structural characteristics with a propensity to fracture (adjusting for age, restorations, endodontics, occlusion, clenching and bruxism), reduce the incidence of tooth fractures? What is the probability that what appears to be early, relatively mild, intermittent episodes of bruxing and clenching will become persistent, aggressive bruxing 
and clenching with major loss of tooth structure and/or fractures? In effect, is there sufficient information available at this time on the natural history of effects of bruxing and clenching on the teeth to provide for evidence-based occlusal therapy on a given individual? What is the risk for implants or complex occlusal restorative treatment of not using stabilization splints to prevent the long term consequences of intermittent or persistent bruxing and clenching, irrespective of whether it is clinically possible to state that bruxing is occurring at a given time? Knowing the risk factors related to occlusal dysfunction and TMD can provide some insight into meaningful cost/benefit/risk formulas needed for cost containment.

\section{Cost/benefit/risk paradigm}

All the paradigms considered thus far have been used as the basis of criticisms relative to over-treatment, or unnecessary dental procedures because they are not evidence-based. In effect, the several paradigm shifts that have been considered briefly here can be focused not only on whether a treatment or procedure is based on acceptable evidence, but on cost effectiveness as well. Therefore, the cost of treatment must be consistent with what is considered to be of true therapeutic value. Unfortunately, at any given time research is better at negating therapies than producing treatment with efficacy, leaving the clinician without a defense for using a therapy for a disorder in which even the cause is unknown. This view suggests a method for cost-containment strategy for third party payers, but not for the clinician.

It has been reported that many forms of physical therapy are incapable of curing or even significantly reducing 'TMD' symptoms better than no treatment. Although some forms of physical therapy are reversible, non-invasive and recommended for 'TMD', why should such therapy be advocated simply because it is better than no therapy? If there is no acceptable evidence that a particular form of physical therapy per se cures or significantly reduces the symptoms of 'TMD', why use it unless it is important to patients' satisfaction. What then is the evidence-based criterion for treatment? There is nothing new about the effect of third party interests in cost/benefit/risk factors on dental practice (Ash, 1985, 1993). Patients' needs may be based on evidence-based data that reflect a cost/ benefit/risk formula consistent with contract limits for an adjusted standard of dental health care.

It seems likely that most of what dentists do could fall under the challenge of the evidence-based paradigm. It is possible that many forms of treatment that have not been established as having efficacy by probabilistic research may ultimately be considered not to be evidence-based treatments and excluded from insurance coverage. The same may be said for procedures that do not satisfy the criteria for being evidencebased. Although such a contingency might appear to be only a reflection of some Health Maintenance Organization (HMO) practices, it may ultimately apply also to fee-for-service contracts where procedures that include the use of a facebow, mounting of casts, centric relation interocclusal records, use of semi-adjustable articulators, and diagnostic 'wax-up' may ultimately require their value to be evidence-based. Recent ideas on cost containment reflect this use of an evidencebased paradigm.

\section{Efficacious/effective therapy paradigm}

By some standards, for treatment to be efficacious it must address the cause of the disease or disorder, not simply provide relief of symptoms as with, for example, from a placebo effect. Therefore, in the absence of a known aetiology, most, if not all, forms of therapy for 'TMD' are palliative, not truly efficacious. Although there is uncertainty in the literature about the apparent efficacy of occlusal therapy for TMD and muscle disorders, there is sufficient evidence to support its effectiveness, e.g. appreciation for the perceived positive changes that occur in myofascial pain from the use of an oral splint (Dao \& Lavigne, 1998). Objections to occlusal adjustment generally relate to it being an irreversible treatment; however, that should not be a consideration for the correction of iatrogenic occlusal interferences that interfere with function.

Repositioning the disk and maintaining it in a 'therapeutic position' (in the case of reducing, anterior disk displacement but not transverse displacement) is thought by some to be at least an effective therapy when occlusal restorative and/or orthodontics are also carried out to help maintain the position of the disk (Summer \& Westesson, 1997). Restricting repositioning therapy to reducing, anterior disk displacement has provided the practitioner with more specific criteria for 
the use of repositioning therapy. In addition, other indications for the use of repositioning therapy relate to failure of conservative therapy, lack of indications for arthrocentesis and lavage, existing need for correction of malocclusion and/or for comprehensive occlusal rehabilitation. Disk repositioning procedures were not recommended by the National Institute of Health Technology Assessment Conference Statement (1996). However, in the presence of persistent pain and failed conservative therapy, such irreversible therapy, as well as surgical approaches (e.g. TMJ arthrocentesis, lavage), become studied options for such patients if they do not have pressing sociomedical problems. There has been a shift away from the importance of the disk in the diagnosis and treatment of 'TMD' (Dolwick \& Dimitroulis, 1996).

Effectiveness of treatment can be expressed in terms of patient-centred outcome criteria, including demand for treatment (Kirveskari et al., 1998). Thus, patientcentred outcomes evidence supports the judicious use of occlusal therapy as effective treatment for some forms of TMD and muscle disorders.

The reduction of the consequences of bruxism and clenching, e.g. excessive wear and fracturing of teeth by the use of stabilization occlusal bite plane splints, can be viewed as reasonably effective occlusal therapy for the prevention of the adverse effects of bruxing and clenching.

Efficacious treatment can include the removal of iatrogenic interferences to occlusal function; the cause of dysfunction is the occlusal interference to function. Also considered to be efficacious therapy, is the correction of periodontal trauma from occlusion. This includes occlusal therapy added to other forms of periodontal therapy used in the treatment of progressive periodontitis where occlusal factors are considered to be one of the aetiologic factors in this form of periodontitis (Svanberg et al., 1995).

In a cost containment era, if a treatment being rendered is considered to satisfy neither the evidencebased nor cause-and-effect paradigms, and therefore not efficacious, a computer generated cost/benefit/risk formula could consider such a treatment invalid and reject the practitioner's claim for services rendered. Assuming that such a scenario might occur in the future, there must be a paradigm shift away from what was considered to be efficacious therapy to one that is effective therapy, i.e. one in which the criteria for a successful outcome is patient-centred. Establishing research-determined patient-centred criteria for effective treatment in the absence of what is considered to be efficacious therapy, provides the clinician with a way to provide evidence-based splint therapy and meet the requirements of cost/benefit/risk paradigms.

If clinical trials have established that a treatment is effective on the basis of acceptable patient-centred criteria, the requirements for an evidence-based treatment should have been met even though the causeand-effect paradigm remains for the scientist to resolve the cause of the disorder being treated and methods to treat it efficaciously. The use of the single or other type diagnostic gold standards previously indicated remains; however, a new gold standard is added, the patientcentred outcomes criteria. Thus, it is possible to have a dual-axis set of criteria for evaluating the success of treatment. However, any research that is carried out indicating that occlusal therapy is effective will be critiqued by a subset of academicians who have their own presuppositions. The only answer is based on well-designed, well-controlled, and well-conducted research. Unfortunately, spurious associations by chance, bias, cofounders, consistency, and many others can be the basis for critiques of any research. That is why those for or against a particular paradigm are able to choose the research findings that favour their own presuppositions. It has occurred in relation to efficacious therapy; therefore, expect it to occur with effective therapy that is based on patient-centred outcome criteria. Described in another way, 'science is not a mechanical process by which observations somehow generate conclusions, but is a battle where ideas [paradigms] compete for acceptance'(Drexler, 1990). When biological certainty is elusive, as is often the case, what is being debated is trans-scientific: a dispute over probabilities, values, desirability, not over facts (Bauer, 1984).

\section{Paradigms and occlusal therapy}

Occlusal interferences are defined here as occlusal contact relations that interfere in a meaningful way with function and/or parafunction (Ash \& Ramfjord, 1995). Although this definition has been related to dysfunction for many years, it does not focus on occlusal interferences as being causal factors in 'TMD' even though it is possible to cite a number of references pointing out that that is the case. Rather the definition 
focuses on an obviously improved potential for occlusal contact relations and patient-centred outcome criteria. Thus, the focus is upon the presence of interferences to function and loci of parafunction, as well as the effect of its removal on the patient's perception of increased function and comfort.

The foregoing definition of occlusal interference does not rule out the use of a broader definition that is used for a comprehensive occlusal adjustment before occlusal rehabilitation, or for prospective, longitudinal studies of occlusal adjustment (Burgett et al., 1992; Kirveskari et al., 1998), i.e. interference to smooth gliding lateral and protrusive movements and interferences to maximal intercuspation in centric relation and centric occlusion.

\section{Removal of occlusal interferences}

Although experimental interferences can cause symptoms such as pain in the muscles and joints, critics who deny that occlusion is an aetiological factor in ' $\mathrm{TMD}^{\prime}$ do not accept such symptoms as being 'TMD'. This is because it is thought that there is insufficient evidence to specify occlusion as any kind of causal factor for 'TMD'. The removal of occlusal interferences as a treatment for 'TMD' is considered by some not to be evidence-based treatment. This view of occlusion has developed partly because of inappropriate forms and degrees of occlusal adjustment, as well as research that does not identify occlusal interferences with the most likelihood of being a co-factor or added factor in some form of temporomandibular and muscle disorder such a chronic traumatic arthritis. Long term prospective studies where all interferences are removed and so maintained, reflect an over-all effect of their removal but do not focus on the short-term effects of more specific forms of interference such as those that obviously and meaningfully interfere with function.

The patient shown in Fig. 1 has an anterior bridge which prevented occlusal contacts with the cuspid during function and bruxing. As shown, there has been fracturing of the porcelain facing on the bridge but still no contact with the cuspid. The patient complained of an inability to 'chew properly': an exacerbation of 'TMD' symptoms. The bridge was remade so that contact with the cuspid in chewing and the 'TMD' symptoms abated, but not immediately. The patient had a prior diagnosis of 'TMD' based on the diagnostic crite- ria of Truelove et al. (1992). Acute and/or chronic trauma to TMJ leads to traumatic arthritis in which the reactive changes are not always totally eliminated with subsidence of the inflammation of the joint tissues.

The question persists: why do some of the symptoms of 'TMD' sometimes abate with an occlusal adjustment of interferences to function? The answers are many, but the most frequent reflex responses include: the data is anecdota; the symptoms would have abated anyway; a placebo effect; it is not really 'TMD'; or simply it is unknown because of the lack of information at the present time on the neuromuscular system. To give any answer reflects some uncertainty, much less to suggest that it is possible to predict the effect of selective occlusal adjustment in a given case. If, for example, an iatrogenic occlusal interference to function is removed by selective grinding and followed very shortly by abatement of the symptoms of 'TMD', is there a possible explanation other than the answers already given? It must be kept in mind that the question is not related to the removal of the cause of the 'TMD' in the first place. It is related to the possibility that by removing the interference the TMJ disk assembly may be able to find a more biologically acceptable position. This kind of relationship is swamped by other factors in epidemiological studies.

It is to be expected that critics require evidence that certain occlusal contact relations interfere with function, and that by removing an iatrogenic occlusal interference the function is improved. An interference to function may be obvious clinically, although adaptation to interferences is often rapid; however, removal of an interference may not be immediately obvious to

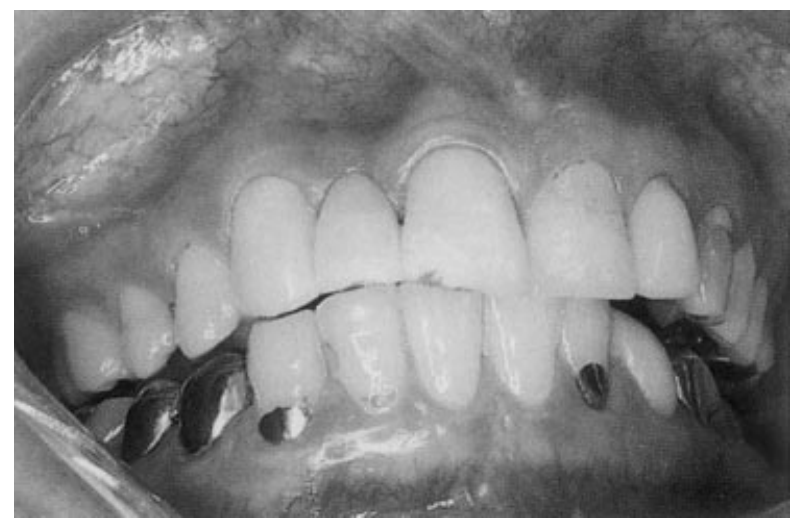

Fig. 1. Occlusal interference to canine function and parafunction as a result of anterior bridge showing fractured porcelain facings. 


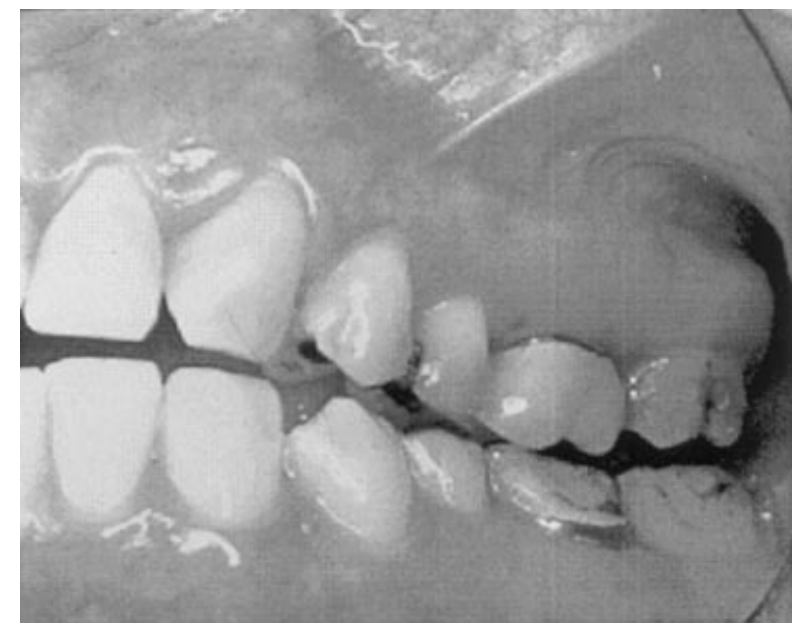

Fig. 2. Occlusal interference to function as a result of crowns on first molars.

the clinician or the patient. It may not be enough to convince the critic of improved jaw movement in chewing using jaw-tracking devices because function can be defined in many ways. However, it is reasonable to relate improved function to patient-centred criteria. Of course, in the first place iatrogenic occlusal interferences should not be placed in the occlusion irrespective of their possible relationship to 'TMD'. The iatrogenic occlusal interference in Fig. 2 was removed with improvement in function and abatement of the symptoms of an exacerbated 'TMD'. The importance of this case is the failure to evaluate the occlusal contact relations for interference to function. Although it was also considered to be an interference to, and an aggravation of, bruxism, that cannot be demonstrated. Nor can the abatement of 'TMD' symptoms be related to cause-andeffect. However, from an educational point of view and clinical reality, iatrogenic occlusal interferences should not be considered as being innocuous. Natural occlusal interferences are no less important but require more detail to consider than the present topic permits.

\section{Bruxism and clenching}

The association between bruxing/clenching and 'TMD' is not thought by some to be a cause-and-effect relationship, and therefore, the use of stabilization splints is not considered to be efficacious, but effective at least for myofascial pain dysfunction. Whatever the reasons for the benefits of such interocclusal devices, which may be collectively expressed as providing some biological advantage for the TMJ, their use continues to be a significant part of conservative therapy for some forms of TMJ and muscle dysfunction, including posttreatment management of repositioning therapy. Discussions over the effectiveness of stabilization splints need to be more focused on patient-centred outcome criteria than on cause-and-effect relationships.

As a researcher in a hospital before the time when it was common to surgically shunt cerebrospinal fluid to the peritoneal cavity or right atrium of the heart in young patients with hydrocephalus, the pervasive sounds of grinding of the teeth by virtually every patient seem to fill the wards. That the cause of bruxing/clenching was not simply a result of the presence of occlusal discrepancies seemed obvious, and whatever the relationship between the occlusion and the central nervous system that was unknown then remains unknown now. However, placement of difficult restorations required careful attention to occlusal interferences, otherwise bruxing was aggravated. Wards filled with bruxing/clenching patients with hydrocephalus are no longer seen, but most every dental office has young and older patients with varying degrees of worn teeth, from that which may be hardly noticeable to that which exceeds anything that might be considered to be a result of normal attrition. Figure 3a illustrates the localized effect of bruxing in a young bruxer; Fig. $3 b$ shows an adult with severe localized bruxing effects; Fig. 3c shows a very early bruxing/ clenching position; and Fig. 3d illustrates very advanced occlusal loss and pulp disease in an older adult.

The dentist's primary concerns for patients, both young and older, is to prevent the consequences of bruxing/clenching. There are no cost/benefit/risk formulas to determine for whom, when, and under what conditions stabilization splints should be prescribed. Resolving these concerns should be given attention until such time as science provides the data for treating the cause of bruxing/clenching, either daytime or night-time bruxing/clenching. The primary approach to cost containment should not begin solely at the delivery site of health care; it should begin with the research site as well, e.g. research on the characteristics of patients with a risk probability for aggressive bruxing/clenching.

Whether or not a patient is bruxing at a specific time may be a moot question except where the diagnosis of 
night-time bruxing and clenching is based on sleep laboratory data ( $\sim 85 \%$ probability) and other clinical examination data (Lobbezoo \& Lavigne, 1997). However, for the clinician undertaking extensive reconstruction there can only be one answer: perform the restorative treatment as if the patient will continue to clench and brux. This approach includes taking into account design principles as well as the use of a stabilization type occlusal bite plane splint. Whether the splint is efficacious for the cause of parafunction is for researchers to answer; whether the splint is effective for the consequence of bruxing and clenching is for the
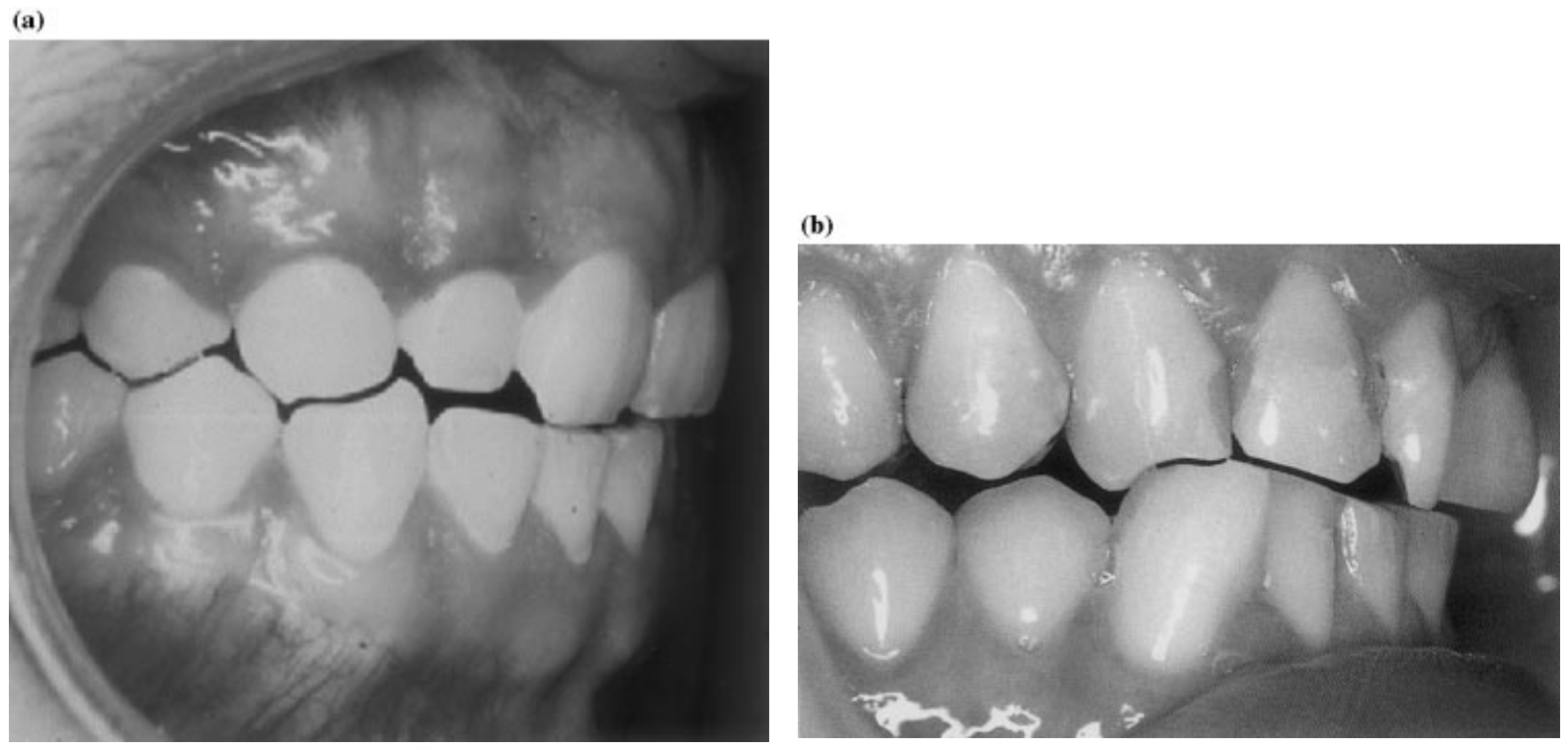

(c)

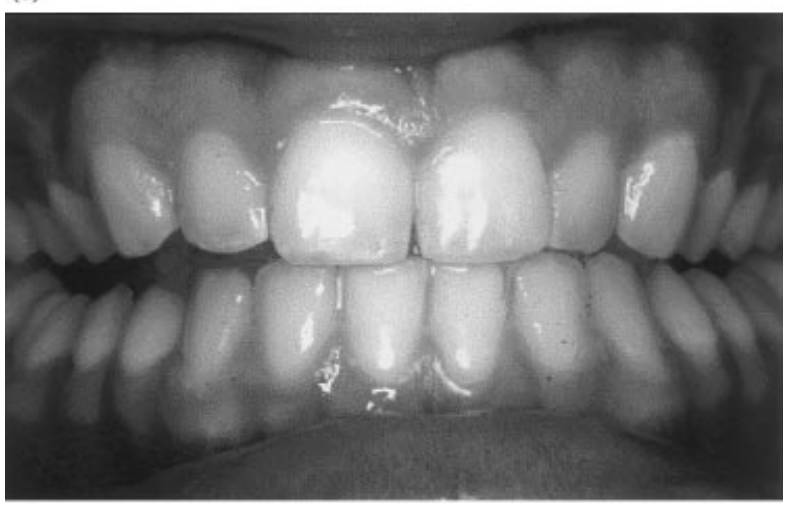

(d)

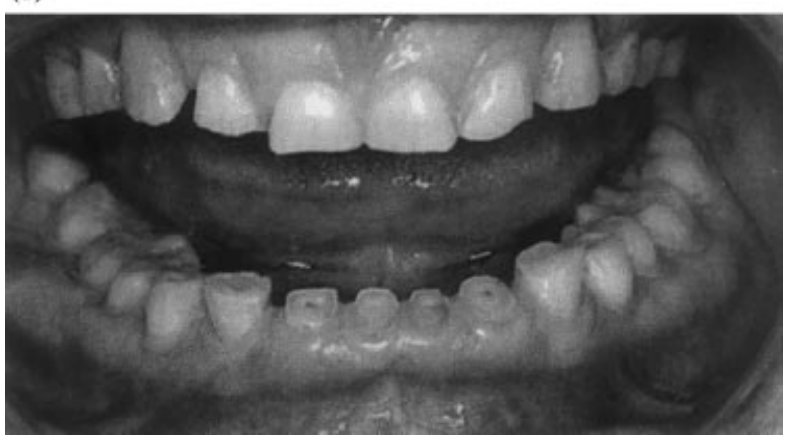

Fig. 3. Bruxism in (a) a young patient; (b), a young adult; (c) a mild case; and (d) an advanced case. 
clinician to answer. From a preventive standpoint, who then will answer the question of when and/or who should receive splint therapy before it becomes obvious that the effects of bruxing have gone too far? Probably not everyone who bruxes and clenches, but for whom shall stabilization type splints be recommended? In order to be evidence-based, is it necessary to undertake sleep laboratory studies in addition to complementary diagnostic clinical criteria (Lavigne et al., 1996) to justify the design of comprehensive restorative treatment and the follow-up use of stabilization splints? The blending of cost/benefit/risk and evidence-based paradigms may have been suggested in a yield/input/ payoff paradigm (Stohler, 1997). Cost containment is not necessarily based on the shifting sands of local standards of care (Selbst, 1997). As already suggested, patient-centred outcomes criteria and the experiential judgment of the practitioner should justify any cost/ benefit/risk paradigm.

\section{Dental education}

The controversy over whether or not occlusion has a role in 'TMD' has overshadowed the importance of occlusion in all other aspects of dental practice, at least in the field of dental education where courses in occlusion have become all but non-existent in some universities and colleges. This problem is reflected in examination scores of graduates on regional and state dental examining boards. Occlusal interferences, both natural and iatrogenic, appear to be thought of as inconsequential even though they obviously interfere with function. It is time that the importance of occlusion in the dental curriculum and in dental practice be reconsidered in light of function, as well as patients' satisfaction and well being. It does not mean that occlusion may not be one of many causal factors in 'TMD', trauma from occlusion, and progressive periodontitis.

\section{Science and paradigms}

Various paradigms have been considered but little has been said of science, at least about the spirit of science, which should be the basis of all paradigms if they are to be considered as being scientific. As already implied, debates over questions about the validity of occlusal therapy for 'TMD' and occlusal dysfunction can be productive. With the exception of the Faustian bargain that science has made with governmental funding agencies, for the most part the ethos of clinicians and researchers is based on norms consistent with professionalism. With these norms, it is possible to view paradigmatic shifts as time dependent necessities of scientific progress rather than theory-laden ideologies.

\section{Conclusion}

Paradigmatic shifts in the management of 'TMD' are not simply reflections of the semantic differences between the studied meanings of efficacious and effective occlusal therapy, but rather a change in the concept of science in the paradigm lost: the 'art and science' of dentistry. However, the biomechanical basis for the practice of dentistry remains seemingly unchallenged.

More emphasis should be placed on patient-centred criteria of what is perceived to be important to patients' function, satisfaction, and needs, as well as dentists' views of what is significant for improvement in dental health.

Occlusal therapy has a number of roles in dental practice. The following are only a few, including the removal of interferences to function, the reduction of periodontal occlusal trauma, and the control (to the extent possible) of the effects of bruxing and clenching on the natural teeth, restorations, implant systems, and on the TMJ. These important aspects of occlusion should not be lost in controversial paradigmatic issues that are not in the best interests of dentistry.

\section{References}

Agency for Health Care Policy and Research (1992) Acute Pain Management: Operative or Medical Procedures and Trauma. Agency for Health Care Policy and Research Publications, Rockville, MD.

OKeson, J.P. (ED.) (1996) Orofacial Pain, Guidelines for Assessment, Diagnosis, and Management. Quintessence, Chicago, IL.

Anderson, H.C. (1974) The Complete Fairy Tales and Stories. (Trans. of original 1874 Danish edn by E.C. Haugaard), pp. 77-81. Doubleday, New York.

Andersen, H.C. (1997) The Emperor's New Clothes. (Trans. by N. Lewis). Candlewick Press, Cambridge MA.

Ash, M.M. \& RAMFJoRD, S.P. (1995) Occlusion, 4th edn. Saunders, Philadelphia, PA.

AsH, M.M. (1993) Bedarfsorientierte praxis der functionslehre [über Konsequenzen einer angemessenen Kosten/Knutzen-Relation]. Phillip Journal, 4, 143. 
AsH, M.M. (1985) Kaufunktion und behandlungsziele-wie viele zahne braucht de mensch? Phillip Journal, 4, 233.

Bauer, H. (1984) Beyond Velikovsky. University of Illinois Press, Urbana, IL.

Bellamy, N. (1999) Clinical trials design: structure modifying agents for osteoarthritis. Future guidelines: areas for development. Osteoarthritis $\theta$ Cartilage, 7, 424.

Beyron, H.L. (1954) Occlusal change in adult dentition. Journal of the American Dental Association, 48, 674.

Blackmore, S. (1999) The Meme Machine. Oxford University Press, Oxford.

BoA, A. (1998) On evidence-based medicine. CMAJ. JAMC, 159, 758.

Burgett, F.G., Ramfjord, S.P., Nissle, R.R., Morrison, E.C., Charbeneau, T.D. \& CAffesse, R.G. (1992) A randomized trial of occlusal adjustment in the treatment of periodontitis patients. Journal of Clinical Periodontology, 19, 381.

ButTs, R.E. (1995) Theory-Laden. In: The Cambridge Dictionary of Philosophy (ed. R. Audi), pp. 797. Cambridge University Press, Cambridge, UK.

CASTI, J.L. (1989) Paradigms Lost: Images of Man in the Mirror of Science, 39. Avon Books, New York.

Chalmer, T.C., Smith, H. Jr., Blackburn, B., Silverman, B., Schroeder, B., Reitman, D. \& Ambroz, A. (1981) A method of assessing the quality of a randomized clinical trial. Controlled Clinical Trials, 2, 31.

Dahl, B.L., Krogstad, B.S., OgaArd, B. \& Echersberg, T. (1989) Differences in functional variables, fillings, and tooth wear in two groups of 19-year-old individuals. Acta Odontologica Scandinavica, 47, 35 .

DAO, T.T. \& LAVIGNE, G.J. (1998) Oral splints: the crutches for temporomandibular disorders and bruxism? Critical Reviews of Oral Biology $\theta$ Medicine, 9, 345.

DAvidoff, F. (1999) Standing statistics right side up. Annals of Internal Medicine, 130, 1019.

DAvies, B.J. (1999) Cost containment mechanisms in Canada. Croatian Medical Journal, 40, 287.

DAvies, H.T. \& CRombie, I.K. (1997) Interpreting health outcomes. Journal of Evaluation of Clinical Practice, 3, 187.

Dawkins, R. (1989) The Selfish Gene, 192. Oxford University Press, Oxford

DeBoever, J.A. (1973) Functional disturbances of the temporomandibular joints. Oral Science Review, 2, 100.

Dolwick, M.F. \& Dimitroulis, G. (1996) A re-evaluation of the importance of the disk position in temporomandibular disorders. Australian Dental Journal, 41, 184.

DreXLeR, K.E. (1990) Engines of Creation [The Coming Era of Nanotechnology], 42. Doubleday, New York.

DWorkin, S.F. \& LeRESCHE, L. (1992) Research diagnostic criteria for temporomandibular disorders: review, criteria, examination and specifications, critiques. Journal of Craniomandibular Disorders Facial Oral Pain, 6, 327.

EveritT, B.S. (1998) The Cambridge Dictionary of Statistics, p. 28 \& p. 211. Cambridge University Press, Cambridge, UK.

Feine, J.S., Widmer, C.G. \& Lund, J.P. (1997) Physical therapy: a critique. Oral Surgery, Oral Medicine, Oral Pathology, Radiology Endodontics, 83, 123.
Feinstein, A.R. (1983a) An additional basic science for clinical medicine: I. The constraining fundamental paradigms. Annals of Internal Medicine, 99, 393.

Feinstein, A.R. (1983b) An additional basic science for clinical medicine: II. The limitations of randomized trials. Annals of Internal Medicine, 99, 544.

Feinstein, A.R. (1983c) An additional basic science for clinical medicine: III. The challenges of comparison and measurement. Annals of Internal Medicine, 99, 705.

Feinstein, A.R. (1983d) An additional basic science for clinical medicine: IV. The development of clinimetrics. Annals of Internal Medicine, 99, 843.

FEUSSNER, J.R. (1996) Evidence-based medicine: new priority for an old paradigm. Journal of Bone and Mineral Research, 11, 877.

FRICtON, J.R. \& SCHIFFMAN, E.L. (1987) The craniomandibular index: validity. Journal of Prosthetic Dentistry, 58, 222.

Gerszten, P.C. (1999) Outcomes research: a review. Neurosurgery, 44, 919.

Goodman, S.N. (1999a) Toward evidence-based statistics. 1. The P value fallacy. Annals of Internal Medicine, 130, 995.

Goodman, S.N. (1999b) Toward evidence-based statistics. 2. The Bayes factor. Annals of Internal Medicine, 130, 1005.

Hugoson, A., Ekfeldt, A., Hallonsten, A.-L., Helkimo, M., Koch, G. \& Nyman, J.-E. (1985) Occlusal wear in children and adolescents (The Jönköping Dental Study) [abstract 43]. Swedish Dental Journal, 9, 274.

Hugoson, A., Bergendal, T., Ekfeldt, A. \& Helkimo, M. (1988) Prevalence and severity of incisal and occlusal tooth wear in an adult Swedish population. Acta Odontologica Scandinavica, 46, 255.

International Headache Society (1988) Classification and Diagnostic Criteria for Headache Disorders-Cranial Neuralgia and Facial Pain, Cephalalgia, 8, (Suppl. 7) 1.

JAKOBSEN, J.R. (1999) A survey of statistical methods used in dental literature. Journal of Dental Education, 63, 350.

KafKA, F. (1954) The Castle. Knopf, New York.

Kirveskari, P. \& Alanen, P. (1999) Emperor's new clothes on occlusion and TMD. Journal of Craniomandibular Practice, 17, 151.

Kirveskari, P., Jäsmä, T. \& Alanen, P. (1998) Occlusal adjustment and the incidence of demand for temporomandibular disorder treatment. Journal of Prosthetic Dentistry, 79, 151.

Kunn, T.S. (1996) The Structure of the Scientific Revolution, First edition, 1962. University of Chicago Press, Chicago, IL.

Lobbezoo, F. \& LAvigne, G.J. (1997) Do bruxism and temporomandibular disorders have a cause-and-effect relationship? Journal of Orofacial Pain, 11, 15.

Lavigne, G.J., Rompré, P.H. \& Montplaisir, J.Y. (1996) Sleep bruxism: validity of clinical research diagnostic criteria in controlled polysomnographic study. Journal of Dental Research, 75, 546.

Lundeen, T.F., Levitt, S.R. \& McKinney, M.W. (1986) Discriminative ability of the TMJ scale: age and gender differences. Journal of Prosthetic Dentistry, 56, 84.

Merskey, H. \& Bogduk, N. (1994) Temporomandibular pain and dysfunction syndrome. In: Classification of Chronic Pain: Descriptions of Chronic Pain Syndromes and Definitions of Pain Terms (eds H. Merskey \& N. Bogduk). IASP Press, Seattle, WA. 
Molnar, S., McKee, J.K. \& Preston, C.B. (1983) Tooth wear rates among contemporary Australian aborigines. Journal of Dental Research, 62, 562.

Moore, D.S. (1997) Bayes for beginners? Some reasons to hesitate. American Statistician, 51, 254.

National Institute of Health Technology Assessment ConferenCE Statement (1996) Management of temporomandibular disorders. Journal of the American Dental Association, 127, 15951603.

Niederman, R. (1998) The methods of evidence-based dentistry. Quintessence International, 29, 811.

NoRmand, S.L. (1999) Meta-analysis: formulating, evaluating, combining, and reporting. Statistics in Medicine, 18, 321.

Palla, S. (1997) Occlusal considerations in complete dentures. In: Science and Practice of Occlusion (ed. C. McNeill). Quintessence, Chicago, IL.

Palmer, A.J. \& Sendi, P.P. (1999) Meta-analysis in oral health care. Oral Surgery, Oral Medicine, Oral Pathology, Radiology Endodontics, 87, 135.

PeAt, D.F. \& BRIGGS, J. (1984) Looking Glass Universe: The Emerging Science of Wholeness. Simon \& Schuster, Boston, MA.

Pincus, T. \& STEIN, C.M. (1999) ACR20: clinical or statistical significance? Arthritis and Rheumatism, 42, 1572.

RAPHAEL, K. \& MARBACH, J.J. JR (1997) Evidence-based care of musculoskeletal facial pain. Journal of American Dental Association, 128, 73.

RASPE, H. (1996) Evidence-based medicine: popular nonsense, old wine in new bottles, or current necessity? Zeitschrift für Arzliche Fortbildung (Jena), 90, 553.

Rothman, R.J. (1986) Modern Epidemiology, 10. Little Brown, Boston, MA

Rothman, T. \& Sudarsham, G. (1998) Doubt and Certainty, 200. Perseus (Helix) Books, Reading, MA.

RuCKer, R. (1982) Infinity and the Mind. pp. 165. Harvester, Sussex.
Sackett, D.L., Rosenberg, W.M.C., Gray, J.A.M., Haynes, R.B. \& RICHARDSON, W.S. (1996) Evidence-based medicine. What it is and what it isn't. British Medical Journal, 312, 71.

Selbst, A.G. (1997) Standard of care: So who decides? [Guest editorial]. Oral Surgery, Oral Medicine, Oral Pathology, Radiology Endodontics, 83, 637.

Seligman, D.A. \& Pullinger, A.G. (1995) The degree to which dental attrition in modern society is a function of age and of canine contact. Journal of Orofacial Pain, 3, 266.

SpiLker, B. (1991) Guide to Clinical Trials. Raven Press, New York. StAlLARD H. (1965) Forty years of gnathology. Oral Rehabilitation and Occlusion Vol II (ed. B.W. Pavone), pp. 1-9. University of California, San Francisco, CA.

StohleR, C.S. (1997) Clinical decision-making in occlusion: a paradigm shift. In: Science and Practice of Occlusion (ed. C. McNeill). Quintessence, Chicago, IL.

Storey, A.T. (1994) Controversies in TMD: riddles of taxonomy. Journal of Orofacial Pain, 8, 333.

Summer, J.D. \& Westesson, P.-L. (1997) Mandibular repositioning can be effective in the treatment of reducing TMJ disk displacement. A long term clinical and MR imaging follow-up. Cranio, 15, 107.

Svanberg, K., Gregory, J.K. \& Gibis, C.H. (1995) Occlusal considerations in periodontology. Periodontology 2000, 9, 106.

Tanenbaum, S.J. (1999) Evidence and expertise: the challenge of the outcomes movement to medical professionalism. Academic Medicine, 74, 757.

Truelove, E.L., Sommers, E.E., LeResche, L., Dworkin, S.F. \& Von KorfF, M. (1992) Clinical diagnostic criteria for TMD. Journal of American Dental Association, 123, 47.

Correspondence: M. M. Ash, University of Michigan, School of Dentistry, University of Michigan, Ann Arbor, MI 48109-1078, U.S.A. E-mail: mmash@umich.edu 